\title{
REFERENCE MODELS FOR SUPPLY CHAIN DESIGN AND CONFIGURATION
}

\author{
Markus Rabe \\ Frank-Walter Jaekel \\ Heiko Weinaug \\ Corporate Management Division \\ Fraunhofer Institute for \\ Production Systems and Design Technology, IPK \\ Pascalstr. 8-9, 10587 Berlin, GERMANY
}

\begin{abstract}
Today more and more essential processes are conducted across enterprise borders, inducing additional challenges in terms of different languages, process types and ontology. Business Process Modelling (BPM) and Simulation are well-understood methods to analyze and optimize the processes within an enterprise. However, they can also be used for cross-organizational application, especially if they are combined with reference structures. This paper explains techniques which support cross-enterprise design and configuration based on Reference Models. Thereby, different approaches such as SCOR, the Integrated Enterprise Modelling (IEM) and a specific Distributed Simulation Method are used and integrated into a consistent Reference Model approach. The application of this approach is illustrated with different projects which each focus on a specific aspect of the supply chain design and configuration.
\end{abstract}

\section{MOTIVATION}

Today, final customers require "better" industrial products like cars, household appliances or electronic devices in more and more short periods. By "better" products the business partners mostly understand the addition of new functionalities and techniques or the enlargement of the performance and capacity. Therefore, during the last decades manufacturers have continuously tended to produce more complex products in a broader set of variants.

Usually, this fact directly relates to the number of subparts and components within the final products as well as to the number of required manufacturing steps. Furthermore, the globalization in form of the very potent logistics today offers to enterprises a worldwide production and purchase. This is a chance and a challenge, because both complexity and distance are the factors which bring the supply chain even more in the focus of the companies' interest. Managers have to realize that no longer single en- terprises stand in competition to each other, but complete supply chains are competing. This covers the traditional material flow and logistic-technical aspects but also has to include the interoperable embedding of service functions and service providers (like trade finance) into the analysis and management of enhanced supply networks.

Reference models are effective and powerful instruments to get an overview and deeper understanding of the supply chain processes, to give orientation for re-engineering and optimization, and finally, to configure and control these processes in operation. However, in order to fulfill all these tasks a reference model has to integrate different functionalities at different implementation levels of a supply chain in a unique and consistent approach. For example, the same reference model can be used for crossorganizational business process modeling, analysis, simulation as well as for process and software configuration at any level of a given supply network.

This paper aims to present an approach based on reference models for supply chain design and configuration, including dynamic analysis by discrete event simulation.

\section{CHALLENGES FOR REFERENCE MODELS}

Reference models form a conceptual frame. They aim to increase the efficiency of modeling, to raise the quality level of the developed models, and to improve the reusability of the models. Therefore, they have example characteristics when they give clues for the development of concrete applications. Simultaneously, they have the function to provide a standard for comparison, and therefore form an instrument for a weak-point analysis and an optimization of the present systems. The use of reference models has the advantage to simplify and speed up the modeling process as they facilitate the identification of structures and thus improve the transparency. Furthermore, they offer support for communication and orientation through the creation of a homogeneous terminological basis. 
Reference models include a systematization, e.g. in form of class structures, model templates and a manual which describes the correct and efficient use of the reference models as well as the validity and constraints of the model (Rabe and Mertins 1998). Depending on the application area there might be the need to add further elements.

Related to supply chain management there exist various approaches and attempts for reference models. Some reference approaches can be used for business process modeling and analysis. Examples are the models of Bowersox, Metz and Cooper/Lambert/Pagh (compare to Corsten and Gössinger 2001), the SCM Maturity Model (McCormack and Lockamy 2004), the CPFR Model (Seifert 2004) as well as the Supply Chain Operation Reference Model (SCOR) (Supply Chain Council 2006).

However, these reference models that are mostly related to business processes do not cover all relevant aspects of the business life. They concentrate on specific aspects, for example on the physical material flow and its related processes, on the financial information exchange or on the human behavior. Especially, SCOR is a reference model designed for the effective communication among supply chain partners. It incorporates elements like standard descriptions of processes, standard metrics and best-in-class practices. Furthermore, SCOR has turned out to be a good base for enhanced models (Stich and Weidemann 2002). However, there are also limitations. For example, SCOR does not include sales administration processes, technology development processes, product and process design and development processes, or some post-delivery technical support processes. Furthermore, the SCOR does not explicitly address training, quality, information technology (IT) development and administration. Overall the SCOR scheme covers most of the workflow processes but has very few relation to service provider functionalities. For example, it has few impact to financial services (only capital assets, payment but no trade finance, insurance).

More general approaches are related to supply chain management software like the J. D. Edwards Supply Chain Management or the business reference models from SAP (Stadtler and Kilger 2005).

Further approaches were developed for education and training. The Delft University of Technology provides an object-oriented approach of a supply chain reference library for supply chain training based upon simulation (Verbraeck and van Houten 2005).

As the various reference models do not cover all business aspects and necessities, the challenge is to have an integrative approach which can close the gaps without loosing quality on the specific area of use. However, which are the functionalities and the span of business processes that have to be covered for such a multi-purpose reference model aiming to design and configure supply chains?

First of all, the span should be defined. The reference model is targeted to supply chains. In the logistical view such supply chains start at the most upstream manufacturer and end at the final customer. A reference model approach has to cover all participating enterprises. This includes the order and material workflow of all enterprises on each existing level (tier) of the complete supply chain as well as enterprises which provide support functionalities such as logistic service providers or financial service providers. However, to manage the supply network on the enterprise level is one side of the coin, only. The other side is to offer reference structures to handle the cross-organizational processes.

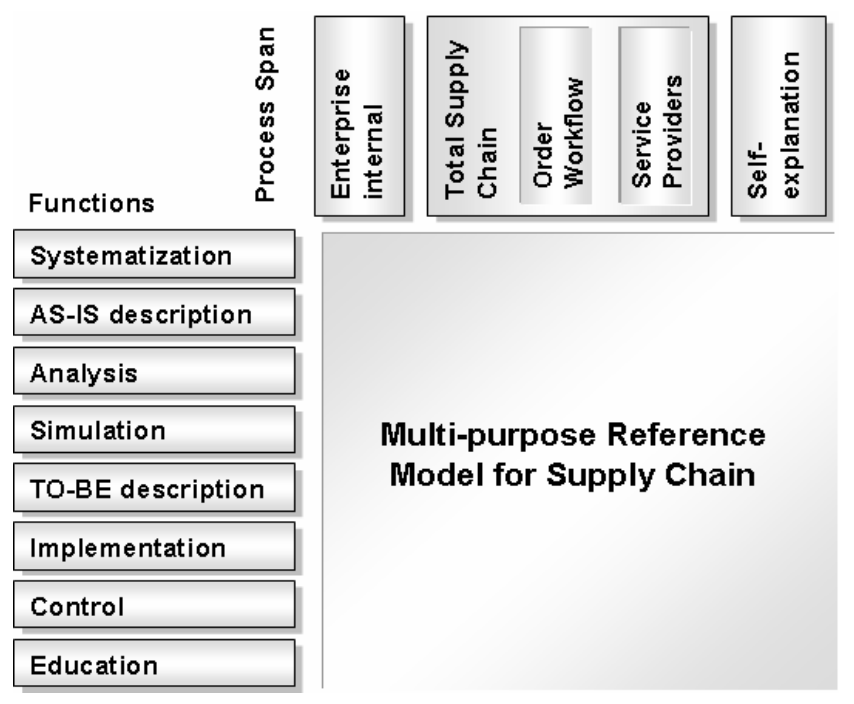

Figure 1: Functionalities and Span of a Reference Model for Supply Chain Design and Configuration

Furthermore, a reference model for supply chain design and configuration has to cover a broad set of consistent, integrated, interoperable and linked functionalities (Figure 1). The same reference approach can be used for a systematization as well as for the evaluation and operation. This helps the user to understand the basic principles of the supply chain and eases the application of the reference model itself. Enhanced by additional explaining information, a didactical concept and an interactive demonstration environment it can be also used for education (see Section 3.5). Obviously, a reference model for supply chain design and configuration requires functions for the description of the as-is situation, for business process analysis (Section 3.2) and the definition of a to-be model (Section 3.3). Simulation is a well defined method for the analysis of complex and dynamic systems. Unfortunately, today business process modeling and simulation are handled quite separately, even if there exist approaches to integrate both spheres (Section 3.1). Hence, the simulation is pointed out as separate function in Figure 1. Finally, the designed and configured processes have to be implemented and controlled during the whole lifecycle. The reference model ap- 
proach has to support also these functionalities. Implementation also means the integration of supporting software tools for supply chain management and execution (Section 3.3 and 3.4).

\section{APPROACH FOR A MULTI-PURPOSE REFERENCE MODEL}

The proposed approach for a reference model for supply chain design and configuration was developed and enhanced systematically and continuously during the last years. Therefore, in the further sub-sections the different functionalities are explained along the development schedule, taking into account application examples from different projects.

\subsection{Basic Concept of a Process-Oriented Reference Model Including Simulation}

The basic design principles for the Business Process Reference Model and its technical embedding within a simulation environment was developed within the IMS project MISSION. The concept is based on the results of the European MISSION Module and on an extension of the Enterprise Modeling tool $\mathrm{MO}^{2} \mathrm{GO}$ (Mertins and Jochem 1999; $<$ http: / /www. moogo. de>).

The general goal of MISSION was to support the Manufacturing System Engineering (MSE) process by integrating tools, which can be used in various aspects of the process, for example tools for process modeling and ofthe-shelf simulation packages. In order to enhance and accelerate the MSE process, they must refer particularly to both, the usage of simulation, and the integration of simulation tools with other software tools (Mertins, Rabe and Jaekel 2000). For the reference process model, the integration of the modeling tools and simulation is of high interest.

The approach includes the modeling aspects which describe how a user can collect the necessary data for the distributed simulation. Furthermore, it describes how the different simulation models can be connected. starting from a template library, via an enterprise model, to the automatic generation of the required interface files. The MISSION approach uses and extends the High Level Architecture approach (HLA) (IEEE 2000; Kuhl, Weatherly and Dahmann 2000) to support the industrial use of distributed simulation.

The core element for the reference model is the template library (Figure 2). This template library allows the definition and management of different templates. Templates are predefined elements for business process modeling, including sub-processes, descriptions, drawings, links to simulation models or further information. The templates are organized in a class structure.

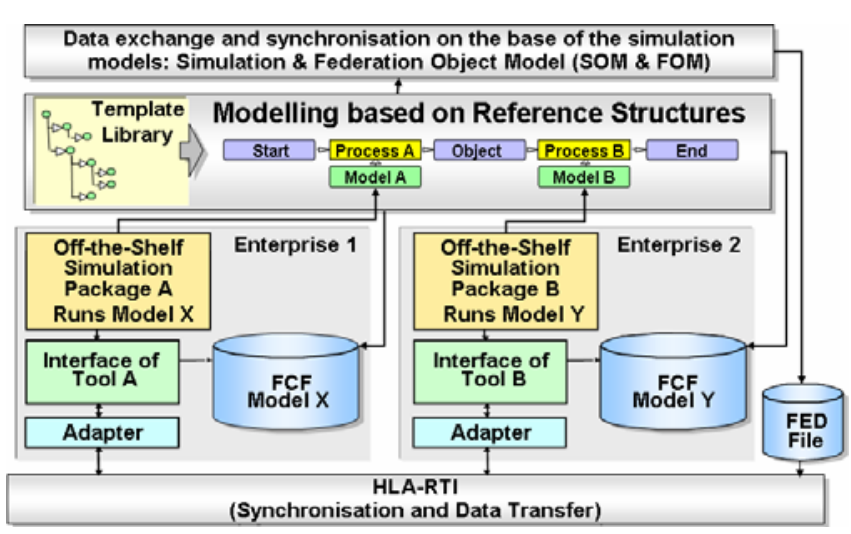

Figure 2: MISSION Project Concept for the Integration of Distributed Modeling and Simulation

The specific "application templates" are directly applicable within a simulation scenario. Each application template refers to a specific simulation model which implements the content of the application template. Each model executes this partial simulation process standalone. Figure 3 gives an overview of the reference model objects and their relations. As the basic set templates and application templates have been developed for 1) supplier (= manufacturer), 2) assembly and warehouse (including modules for headquarter, assembly plant, warehouse, transportation), 3) trading (including the modules distributor, retailer, transportation) and 4) for monitoring and statistics. The application templates cover the basic aspects of a supply chain, describe the exchanged objects (products, orders, information) and can be configured by parameters. These are mainly the same parameters which are used for a supply chain configuration. Therefore, these models can be directly re-used for the specification of the supply chain and its interface requirements (Rabe and Jaekel 2004b).

A simulation manager software supports the definition and the interoperability of simulation templates by exchanging objects (Mertins, Rabe and Jaekel 2000). It delivers a graphical way to design the global business processes and then to derive the te simulation scenario. The simulation manager ensures the consistency between the federate configuration files (FCF) for a distributed simulation scenario by the generation of all FCFs for the federates and the Federate Execution Definition (FED) file for the HLA RTI, automatically (Rabe and Jaekel 2004a).

Summarizing, within the MISSION project the base for the interoperable reference model for supply chain design and configuration was developed and applied to the simulation of globally distributed manufacturing systems. The project has demonstrated that for distributed systems, where different persons perform the modeling task at different locations, reference models can improve the work, significantly (Rabe and Weinaug 2005). However, the MISSION approach has also limitations. The models con- 


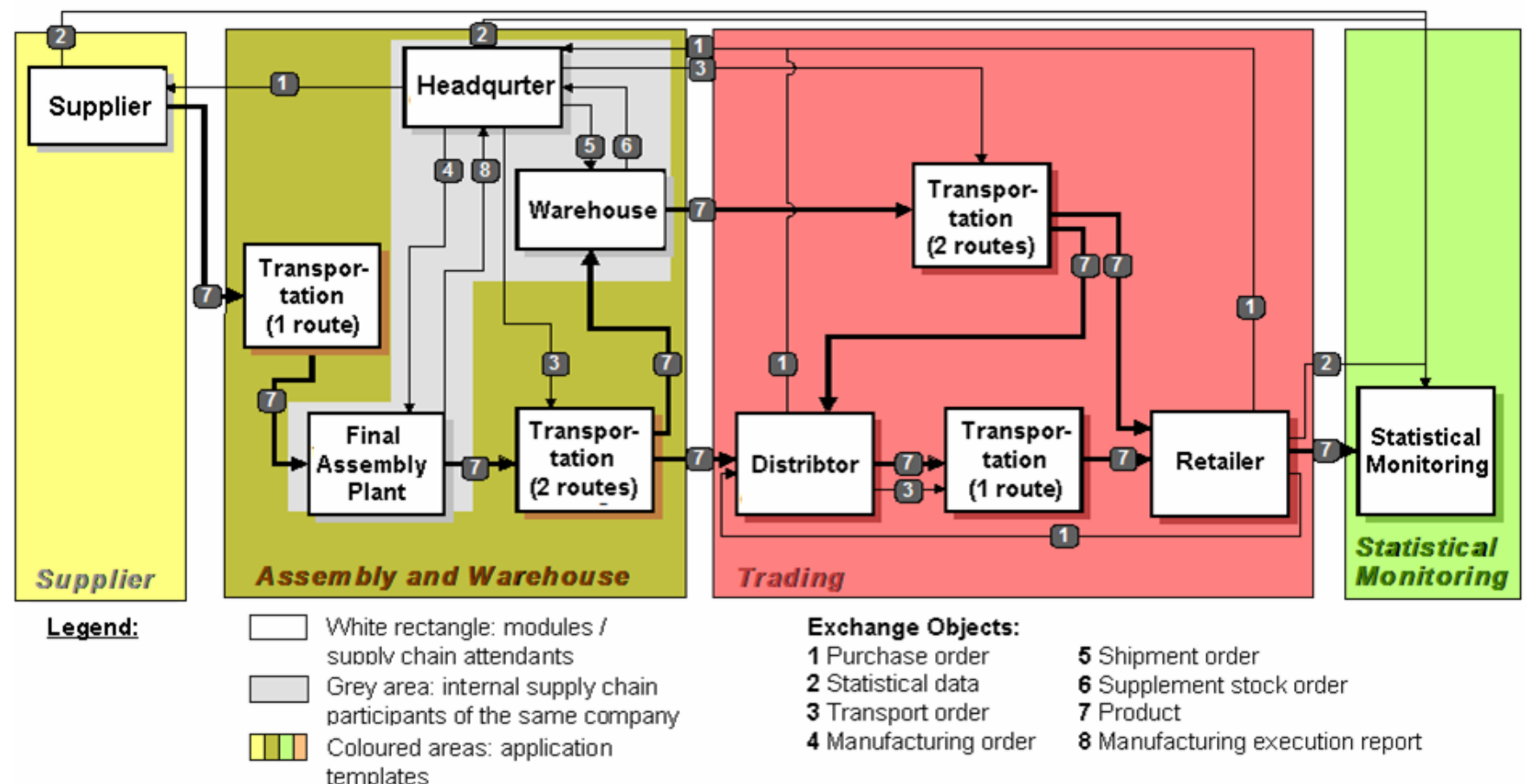

Figure 3: Overview of the Reference Model Objects (Application Templates, Modules, Exchange Objects)—Based on the MISSION Project

centrate on logistic aspects in technical meaning but less on planning, IT-support, control etc. Hence, in further projects the reference model was amended to model the business processes in more accuracy.

\subsection{Reference Model for the Analysis of Distributed Business Processes}

In order to invent and specify new methods and tools for the efficient execution of supply chains in Europe, 10 different enterprises in 3 European supply chains have been investigated in the SPIDER-WIN project. The model was established in order to identify their specific collaboration processes, gaps and improvement potentials, and then to specify the new methods and software requirements on a semiformal, clearly understandable level.

Here reference models can provide a common namespace and structures for helping to compare the different enterprises and supply chains for analyzing the commonalities and differences. A common model sketch in form of different template models and a guideline document suite was developed by Fraunhofer IPK and its R\&D partners, in order to guarantee that all information and detected requirements can be systematically documented within one single, consistent general model and documentation.

Hence, the model sketch consists of a single enterprise template model and a template model for the supply chain (Rabe and Weinaug 2005). Both were based on the use of reference class structures which define common terms for objects and enable overall evaluation procedures of the model. Furthermore, these classes inherit specific attributes which enable to hold in parallel the English name and the name in the native language. These names can be switched, enabling the discussion based on the native terms within the companies, and using the English terms when discussing and comparing the model in the project team.

The single enterprise template model was developed taking into account three objectives. First, the template can be used during interviews (together with the guideline) to ask the interviewee each area of interest and to structure the interview along the process under consideration. The second objective of the template model is a common naming of the process elements. The wording and structure of the template model is orientated at the Supply-Chain Operations Reference-model (SCOR). Third, the template serves as the base for the individual process model of the interviewed company. This individual process model is the core documentation of the interview results. Contrary to the interview protocol this model is structured with respect to the processes as well as to the objects and attributes.

As the modeling of the single enterprise is done based on the reference template, each enterprise within the supply chain is modeled in a similar structure. This increases the comparability of the different models and also enables an easier merge of the models into a general overall model.

Additionally, the template model for the supply chain was developed to support the modeling of the supply network as a whole, thereby connecting the single models. This model shows the relation of different enterprises 
along the supply chain with respect to their exchange of information and goods.

The field studies itself were performed in the regions, separately. The consultants exchanged the models and interview protocols in order to get feedback and to synchronize the models. Furthermore, aspects detected in one region, which could be of importance to other regions were exchanged. Due to the careful preparation, very few physical meetings of the coaches were required.

The three supply networks models have then been merged to one general as-is situation. This general model contains the "standard processes" and identifies possible variants (Rabe and Mussini 2005).

The result is the first step for the customization of a supporting software system for the supply network, identifying the modules required as well as the data and processes to be adapted. This was done in a specific reference model for method and software development and supply chain configuration which is called SPIDER-WIN Supply Chain Management Model (Rabe, Gocev and Weinaug 2006).

\subsection{Reference Model for the Development and Customization of Methods and Software}

The development path of the SPIDER-WIN SCM Model is summarized in Figure 4. Based on the field study results from the different European supply chains which were merged into a General Model, the SPIDER-WIN SCM Model was developed. In contrast to the General Model, the SPIDER-WIN SCM Model represents the definition of a to-be-model.

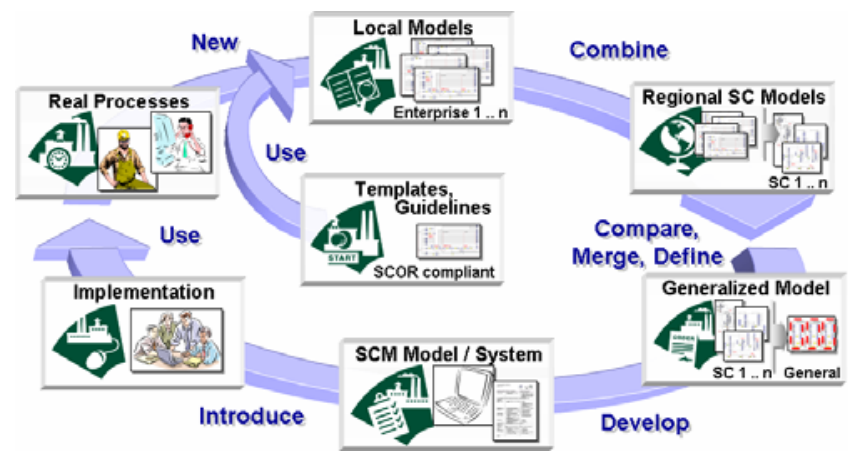

Figure 4: Development Path of the SPIDER-WIN SCM Model

It was created with the objective to have a lean, efficient and flexible model which defines the processes of exemplary companies within a supply chain structure in order to demonstrate how and where the SPIDER-WIN Platform supports these processes. For this purpose the SPIDERWIN SCM Model provides a context-sensitive access to relevant model information. The model consists of a limited but well defined number of base elements and a clear and hierarchical structure of the model. Furthermore, the SPIDER-WIN SCM Model has integrated different standards into one model. The Integrated Enterprise Modelling Method (IEM) was used as basic modeling method, SCOR 7 for a namespace of actions and the XML Common Business Library (xCBL) to support the software development with an object-oriented definition of the information exchange and software functionalities.

The SPIDER-WIN SCM Model enables an integrated visualization of the business processes in the companies and the SPIDER-WIN Platform support of these processes. Hence the SPIDER-WIN SCM Model (1. in figure 5) consist of two major parts. The first part defines the business processes and typical characteristics for each tier in a symbolic reference supply chain (2. in figure 5). For example, a tier 2 supplier has first to select the basic process scheme "tier-2", that includes all basic processes and interface requirements for this tier.

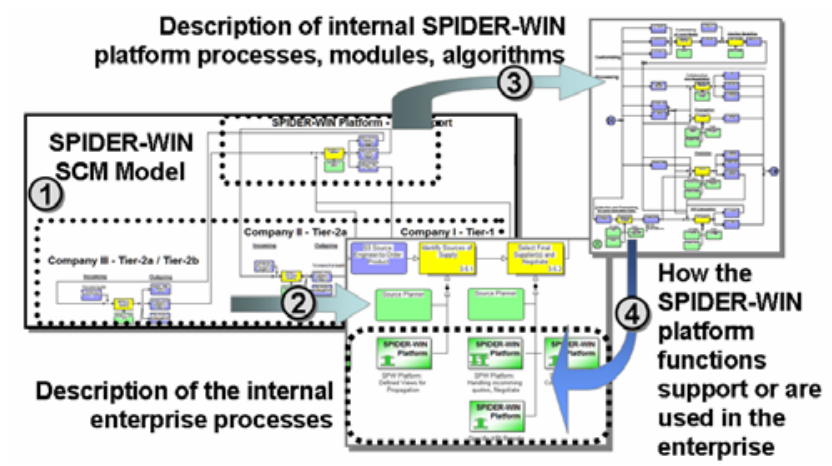

Figure 5: SPIDER-WIN SCM Model Components and Structure

The second part of the SPIDER-WIN SCM Model defines the "SPW Platform" (3. in figure 5) and includes all the associated services (IT infrastructure, software access, customization, maintenance and support), algorithms, basic data relations, component descriptions and the processes to be implemented at the platform.

Both parts are integrated into one common reference model (4. in Figure 5), which integrates the users' (new) business processes and the SPIDER-WIN Platform functionality. Each user company can read the relationships among its processes and the Platform processes, thereby achieving transparency where and how the SPW Platform supports specific processes or which processes exchange information with the platform (Figure 6).

The structure of the SPIDER-WIN SCM Model provides furthermore the process-based support of the implementation and configuration of the supply chain software. This includes the wide range of tasks and processes which have to be performed during the implementation of the Platform in a concrete network. In fact, the greatest advantage of the SPIDER-WIN SCM Model in combination with the Platform is to bring all the various supply chain busi- 
ness partners the first time together on one round table to discuss, negotiate and agree supply chain business process constraints (e.g. time to deliver, service levels, stock limits, etc.).

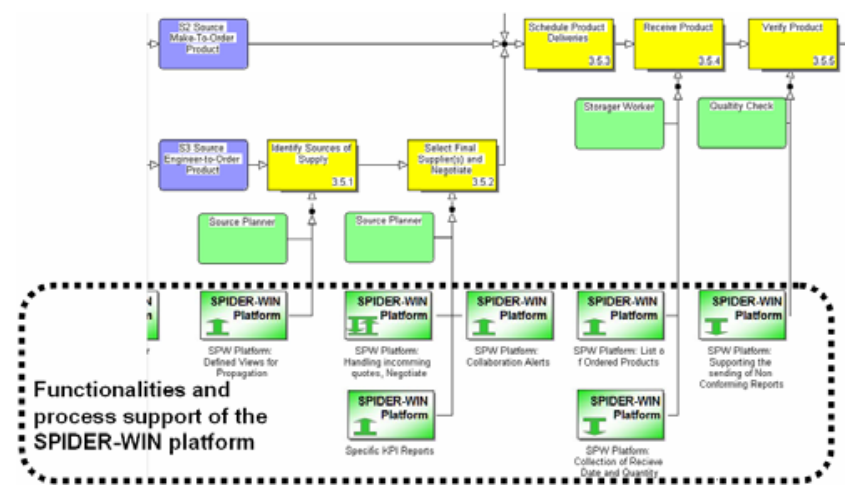

Figure 6: Enterprise Internal Processes (here: level 2) and the Integrated Visualization of their Support by the SPW Platform Functionalities Within the SPIDER-WIN SCM Model

All aspects considered by the SPIDER-WIN SCM Model form an interoperable reference model, including the following major items and features (Rabe, Gocev and Weinaug 2006):

- General description of SCM related business processes

- Support during the development phase of the SPW Platform

- Visualization and description of processes supported by the SPW Platform

- Provision of support during the implementation and introduction phase at end user companies

- Foundation for further improvements of the platform functionalities in the future.

\subsection{Enhanced Supply Chain Reference Model including Service Provider Functionalities}

The SPIDER-WIN SCM reference model mainly is designed and successfully used by small and medium sized enterprises (SME) within manufacturing oriented supply networks. The focus is on the physical material flow and all processes around its technical handling. This is a broad area but also a limitation because it does not cover all optimization possibilities which would be enabled by an integration of service providers and their processes into the reference model concept.

The FLUID-WIN project (FLUID-WIN consortium 2006) targets business-to-business (B2B) manufacturing networks and their interactions with the logistics and financial service providers. More or less the idea is to link one $\mathrm{B} 2 \mathrm{~B}$ network to other $\mathrm{B} 2 \mathrm{~B}$ networks and further to integrate intelligently supportive functionalities from the finance and logistic domain. Hence, the two-dimensional view of a supply chain becomes to a multi-dimensional cognition of the total supply network. The project was started in January 2006 and aims to develop a platform which can seamlessly integrate and transfer data among all the various partners in order to enhance the competitiveness of the whole business sector in Europe and to make the business processes as efficient as possible. For a single company this means that the communication with all business partners (customer, supplier, carrier, bank) will be supported by one unique, integrated environment and will be based on the individual requirements. This will be independent from the communication environment which the business partner uses. Especially, SMEs will benefit from the FLUID-WIN platform, because they have usually many customers and suppliers with their own and incompatible communication environments.

FLUID-WIN requires the enhancement of the reference model concept by the integration of financial and logistic service providers' processes and their crossorganizational collaboration with the manufacturers (Figure 7). The new reference model concept is currently applied for analyzing purposes and will be base for method and software development in the enhanced supply chain configuration, too (comparable to Section 3.3).

The SPIDER-WIN SCM model was used as a starting point for the model, because it excellently describes the manufacturing sector. However, new processes for the financial and logistic domain had to be added. This includes the workflow of the different services from both the providers' side and the clients' side as well as the necessary information exchange. Unfortunately, the SCOR scheme does not help to define a namespace for the new domain because it contains only few descriptions of logistic services and nearly no explanation of financial impacts. Hence, domain experts have described the processes in a first step.

\subsection{Approach of a Supply Chain Demonstrator}

Generally, supply chains are characterized by complexity, randomness, dynamics and missing transparency. However, these are not the real reasons for the problems in the supply chain management but the limited human ability to recognize and handle them. Furthermore, sometimes enterprises are suspicious that delivered information can be used to increase the economic pressure on them (e.g. information relating to free capacity can be used during prize negotiations). Hence, the topic trust impacts the supply chain. If there is no trust in the supply chain, partners might intend to be less transparent. Very often the individual decision is made based on local interests, without taking into account 
or without the knowledge of the consequences for the whole supply chain.

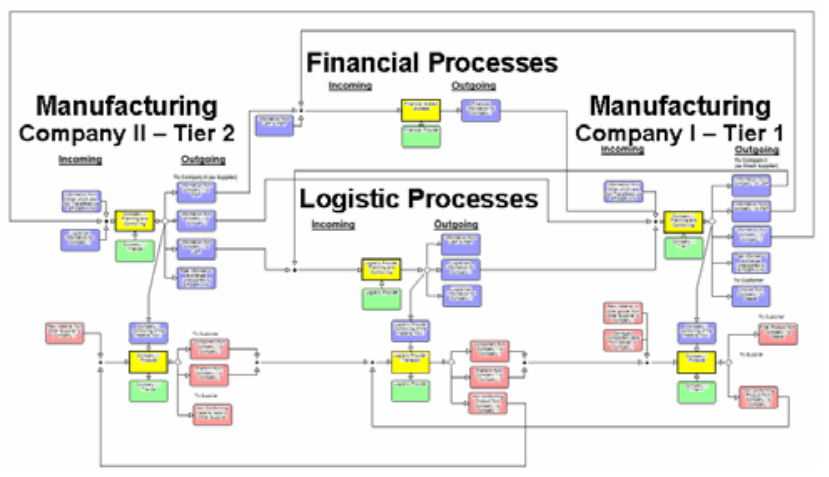

Figure 7: FLUID-WIN Supply Chain Model Including Financial and Logistic Service Providers

Simulation helps to understand the behavior of networks and to study and improve a network as a whole. However, the development of such a network model is complicated, as it comprises several distributed entities and might also be forced to introduce the human-in-the-loop or to include IT control systems in the simulation run. The authors propose a supply chain demonstrator, that can run distributed, combines several (local) simulation models into one federation and also can include non-simulation federates like human user interfaces or additional IT systems.

The Supply Chain Demonstrator is addressed especially to small and medium sized enterprises in order to convince them that a well-balanced supply chain support by specific management and execution mechanisms has significant benefits. In a first step, the Supply Chain Demonstrator visualizes the situation of a supply chain without any supply chain management, where the information exchange appears only bilateral. In a second step it demonstrates how specific management mechanisms or new methods favorably affect the supply chain, and how each partner can participate on these positive effects. And finally the Supply Chain Demonstrator aims to demonstrate the mode of action of IT tools that support the supply chain execution and management, like the SPIDER-WIN Platform (Rabe, Jaekel and Weinaug 2006).

In order to fulfill the aims, the Supply Chain Demonstrator consists at least of 3 logical components:

1. Several workplaces where each participant of the demonstration can "play" a different role within the supply chain (independent decisions but in interaction with the supply chain),

2. A component for the customization of the supply chain and the demonstration scenario, for monitoring the supply chain as well as for the evaluation and visualization of the results,
3. An interface for the integration of IT-oriented supply chain support tools.

Especially in the first two components the reference model approach has strong impacts. The MISSION approach (Section 3.1) provides the methods, techniques and tools to run independent distributed simulation models in one scenario and a configuration mechanism based on business process models. Furthermore, the reference model (Sections 3.3-3.4) provides supply network elements, processes and structures which can be used for the configuration, control and monitoring of the supply chain demonstration. Enhanced by specific scenarios and an education concept a holistic and interoperable Supply Chain Demonstrator will arise.

\section{CONCLUSION}

Companies need instruments to manage their own processes in the context of the supply networks they are part of, including the related service providers. Reference models have proved to be an efficient means for this purpose. Fraunhofer IPK has continuously developed and enhanced a proposal for a unique and consistent concept for business process modeling based on a reference model which integrates various functions. The user, regardless whether manager, consultant, IT responsible person or secretary, has the possibility to access the same reference model and use it for different purposes like analysis, simulation and re-engineering of business processes and implementation of software solution. Currently, the reference model concept is enhanced by a supply chain demonstrator as an education component.

\section{ACKNOWLEDGEMENT}

The European Module of the IMS MISSION project was carried out with financial contribution of the European Commission under the specific RTD Programme, Esprit Project 29 656. The SPIDER-WIN (IST 507 601) and FLUID-WIN (IST 027 083) project are carried out with financial contribution of the European Commission under the 6th Framework Program.

\section{REFERENCES}

Corsten, H., Gössinger, R. 2001. Einführung in das Supply Chain Management. Munich: Oldenbourg Wissenschaftsverlag $\mathrm{GmbH}$.

FLUID-WIN consortium. 2006. FLUID-WIN Deliverable D3 - Project Presentation. Available via $<$ http:// www . fluid-win. de/private/FLW-8-200604-22-IPK-D03_Project_Presentation$1 f \cdot p d f>$ [accessed May 11, 2006]. 
IEEE. 2000. IEEE 1516-2000 Standard for Modeling and Simulation (M\&S) High Level Architecture (HLA) New York: Institute of Electrical and Electronics Engineers, Inc.

Kuhl, F., R. Weatherly, J. Dahmann. 2000. Creating Computer Simulation Systems - An Introduction to the High Level Architecture. New York: Prentice-Hall Inc.

McCormack, K., A. Lockamy III, A. 2004. The development of a supply chain management process maturity model using the concepts of business process orientation. Available via <http://supplychain redesign.com/publications/scm-2004. paf $>$ [accessed July 4, 2006].

Mertins, K., R. Jochem. 1999. Quality-Oriented Design of Business Processes. Boston: Kluwer Academic Publishers.

Mertins, K., M. Rabe, and F.-W. Jaekel. 2000. Neutral Template Libraries for efficient distributed Simulation within a Manufacturing System Engineering Platform. In Proceedings of the 2000 Winter Simulation Conference. Piscataway, New Jersey: Institute of Electrical and Electronics Engineers.

Rabe, M. and K. Mertins. 1998. Reference models of Fraunhofer DZ-SIMPROLOG. Handbook on Architectures of Information Systems, 639-649. Springer.

Rabe, M. and F.-W. Jaekel. 2004a. The MISSION project - demonstration of distributed supply chain simulation. In Enterprise inter- and intra-organizational integration, 235-242. Boston, Dordrecht, London: Kluwer.

Rabe, M., and F.-W.Jaekel. 2004b. Distributed Simulation and Supply Chains. In Proceedings of the International Conference on Competitive Manufacturing 4-6 February 2004 (COMA'04). 437-442. Stellenbosch, South Africa, University of Stellenbosch.

Rabe, M., and H. Weinaug. 2005. Methods for Analysis and Comparison of Supply Chain Processes. In European SMEs. 11th conference on Concurrent Engineering (ICE), Munich 20.-22.06.2005, 69-76.

Rabe, M., and B. Mussini. 2005. Analysis and comparison of supply chain business processes in European SMEs. In European Commission (ed.): Strengthening competitiveness through production networks - A perspective from European ICT research projects in the field 'Enterprise Networking', Luxembourg: Office for Official Publications of the European Communities.

Rabe, M., P. Gocev, and H. Weinaug. 2006. ASP supported execution of multi-tier manufacturing supply chains, In Proceedings of the International Conference on Information Systems, Logistic and Supply Chain ILS'06, Lyon (France), 14-17 May 2006.

Rabe, M., F.-W. Jaekel, and H. Weinaug. 2006. Supply Chain Demonstrator based on Federated Models and HLA Application. In Simulation und Visualisierung
2006, 329-338. Ghent, Erlangen, San Diego, Delft: European Publishing House.

Seifert, D. 2004. Efficient Consumer Response: Supply Chain Management (SCM), Category Management (CM) and Collaborative Planning, Forecasting and Replenishment (CPFR) als neue Strategieansätze. Mering: Verlag Hampp.

Spur, G., K. Mertins, and R. Jochem. 1996. Integrated Enterprise Modelling Method. Berlin: Beuth Verlag.

Stadtler, H. and Ch. Kilger. (ed.) 2005. Supply Chain Management and Advanced Planning. Concepts, Models, Software and Case Studies. Berlin: Springer.

Stich, V., and M. Weidemann. 2002. Decision support for improvement of logistics performance in production networks. In Challenges and Achievements in Ebusiness and E-work, 638-645. Amsterdam.

Supply-Chain Council. 2006. SCOR Homepage. Available via <http : / / www . supply-chain. org >.

van Houten, S.-P., A. Verbraeck, S. Boyson, and T. Corsi. 2005. Training for today's supply chains: An introduction to the distributor game. In Proceedings of the 2005 Winter Simulation Conference. Piscataway, New Jersey: Institute of Electrical and Electronics Engineers.

Verbraeck, A. and S.-P. van Houten. 2005. From simulation to gaming: An object-oriented supply chain training library. In Proceedings of the 2005 Winter Simulation Conference. Piscataway, New Jersey: Institute of Electrical and Electronics Engineers.

\section{AUTHOR BIOGRAPHIES}

MARKUS RABE is head of the corporate logistics and processes department of Fraunhofer IPK. He is responsible for business process planning, factory planning and simulation and head of the Berlin Demonstration Center for Simulation in Production and Logistics. He is member of several conference program committees and was chair of the conference "Simulation in Production and Logistics" in 1998, 2000 and 2004. More than 120 publications and editions report from his work. His e-mail address is <markus.rabeaipk. fraunhofer.de> and the Web address is $<$ http: / / www . um. ipk. fraunhofer . de/en>.

HEIKO WEINAUG studied industrial engineering with the technical orientation of mechanical engineering and logistics at the Technical University of Berlin. Since 2000 he is with the Fraunhofer IPK. He has contributed to several national and international research and consulting projects with focus on simulation, business process reengineering in business as well as public sector and implementation of new analyzing methods and reference models. His e-mail address is <heiko.weinaugaipk.fraunhofer . de>. 